\title{
Scalar dipole dynamical polarizabilities from real Compton scattering data
}

\author{
S. Sconfietti ${ }^{1,2, *}$, B. Pasquini ${ }^{1,2}$, and P. Pedroni ${ }^{2}$ \\ ${ }^{1}$ Dipartimento di Fisica, Università degli Studi di Pavia, 27100 Pavia, Italy \\ ${ }^{2}$ Istituto Nazionale di Fisica Nucleare, Sezione di Pavia, 27100 Pavia, Italy
}

\begin{abstract}
We present an extraction of the scalar dipole dynamical polarizabilities from proton real Compton scattering (RCS) data below pion-production threshold. The theoretical approach relies on dispersion relations, and on the low-energy expansion and multipole expansion of the scattering amplitude. The statistical analysis is based on the parametric bootstrap technique, that revealed to be crucial to deal with problems inherent to both the low sensitivity of the RCS cross section to the energy dependence of the dynamical polarizabilities and the poor accuracy of the available data sets.
\end{abstract}

\section{Theoretical framework}

The real Compton scattering (RCS) amplitude can be separated into a Born contribution, describing the scattering off a pointlike nucleon with anomalous magnetic moment, and a structure-dependent part referred as non-Born term. In the low-energy expansion (LEX) of the non-Born term, the leading-order effects are given by static polarizabilities, that are defined in the limit of zero frequency of the photon field and therefore measure the response to a static external electromagnetic field. The response of the nucleon internal degrees of freedom to an external, real-photon field of arbitrary energy is instead parametrized by dynamical polarizabilities [1-4]. They are functions of the excitation energy and encode the dispersive effects of $\pi N, N^{*}$ and other higher-energy intermediate states.

Dynamical polarizabilities are defined through the multipole expansion of the RCS amplitude in the center-of-mass $(\mathrm{cm})$ frame $[1,5-7]$. In particular, for the scalar DDPs one has:

$$
\alpha_{E 1}(\omega)=\frac{2 \bar{f}_{E E}^{1+}+\bar{f}_{E E}^{1-}}{\omega^{2}}, \quad \beta_{M 1}(\omega)=\frac{2 \bar{f}_{M M}^{1+}+\bar{f}_{M M}^{1-}}{\omega^{2}},
$$

where $\omega$ is the photon cm energy. In Eq. (1) the multipole amplitudes $f_{T T^{\prime}}^{l \pm}$, with $T, T^{\prime}=$ $E, M$, correspond to transitions $T l \rightarrow T^{\prime} l^{\prime}$, the superscript indicates the angular momentum $l$ $\left(l^{\prime}=l \pm\{1,0\}\right)$ of the initial (final) photon and the total angular momentum $j=l \pm 1 / 2$, and $\bar{f}$ indicates the non-Born contribution to the multipoles.

In the following, we will discuss a possible strategy to access the scalar DDPs from data for the RCS unpolarized cross section below pion-production threshold. The Born contribution to the cross section is calculated exactly in terms of pole diagrams involving a single

\footnotetext{
*e-mail: stefano.sconfietti@pv.infn.it
} 
nucleon exchanged in the $s$ - and $u$-channels and $\gamma N N$ vertices taken in the on-shell regime [1]. For the non-Born part, we use the multipole expansion. Thanks to the fast convergence of the multipole expansion of the unpolarized cross section at low energy, we can restrict ourselves to the contribution from the multipole amplitudes up to $l=3$. Then, the scalar DDPs are fitted to the data, while the remaining contributions are calculated through fixed- $t$ subtracted DRs $[8,9]$. The dispersion calculation is performed in terms of six independent invariant amplitudes $A_{i}, i=1, \ldots, 6$, which can be recast in terms of the multipole amplitudes $f_{T T^{\prime}}^{l \pm}$ as explained in App. A of Ref. [3]. The six invariant amplitudes are obtained from subtracted dispersion integrals in both the $s$ and $t$ channels, and subtraction constants. The subtracted integrals are saturated by $\pi N, \pi \pi N$ and heavier-meson intermediate states in the $s$ channel, and $\pi \pi$ intermediate states in the $t$ channel [8]. In our work, the input of the $s$-channel integrals for the pion-photoproduction amplitudes is taken from MAID [10]. The calculation of the contributions beyond $\pi N$ in the $s$-channel and of the $t$-channel integrals is performed according to $[8,9]$. The subtraction constants are given by the static leading-order scalar and spin polarizabilities. For the scalar DDPs, we perform a LEX up to the fifth order in the $\mathrm{cm}$ photon energy, and we take into account the residual contribution beyond the LEX through two energy-dependent functions fitted to the predictions of DRs. The zero-energy limit of the LEX is given by the static dipole scalar polarizabilities, while higher-order terms represent dispersive contributions and recoil corrections given in terms of static leading and higher-order scalar and spin polarizabilities. In our fit, we take as free parameters the polarizability difference $\alpha_{E 1}-\beta_{M 1}$ and the dispersive polarizabilities $\alpha_{E 1, v}$ and $\beta_{M 1, v}$ [9]. The polarizability sum is fixed by the Baldin's sum rule, smeared according to its resolution, i.e. $\alpha_{E 1}+\beta_{M 1}=(13.8 \pm 0.4) 10^{-4} \mathrm{fm}^{3}$ [11]. Furthermore, the static spin polarizabilities are taken from the experimental values of Ref. [12] and the remaining higher-order scalar and spin static polarizabilities are taken from the predictions of DRs $[9,13]$.

\section{Fitting strategy}

The very low sensitivity of the data to the higher-order dispersive coefficients of the scalar DDPs causes high correlations among the fitting parameters. In order to face this problem, we used a combination of the simplex [14] (that is a purely geometric minimization algorithm) and the parametric bootstrap (that is a Monte Carlo technique) methods. Each bootstrap "measurement" is assumed to be Gaussian distributed around a given experimental data point with a standard deviation given by its statistical error. All bootstrapped points of a given subset are then shifted by the same quantity proportional to the published systematic error, assumed to be uniformly distributed. If we define as cycle a number of bootstrapped points equal to the total number of points in the considered experimental data set, the bootstrap sampling can be described as:

$$
\mathcal{S}_{i, k, j}=\left(1+\delta_{k, j}\right)\left[\mathcal{S}_{i}^{\exp }+\gamma_{i, j} \sigma_{i}^{\exp }\right]
$$

where $\mathcal{S}_{i}^{\exp }$ stands for the experimental value of the differential cross section, together with its statistical error $\sigma_{i}^{\text {exp }}$. In Eq. (2), the index $i$ runs over the data points in the whole set, the index $k$ labels each subset, and the index $j$ indicates the bootstrap cycle. Furthermore, the random numbers $\delta_{k, j}$ are uniformly distributed as $\mathcal{U}\left[-\Delta_{k}, \Delta_{k}\right]$, where $\pm \Delta_{k}$ are the published systematic errors, while the numbers $\gamma_{i, j}$ are sampled from the standard Gaussian distribution $\mathcal{N}[0,1]$. When different systematic-error sources are quantified, $\delta_{k, j}$ is the combination of all the contributions. The minimization is performed after a complete cycle, and the output for the fitted values of the polarizabilities is stored. Repeating the bootstrap cycle a very large $n_{R}$ number of replicas (we choose $n_{R}=10000$ ), we are finally able to reconstruct the probability distributions for every fitted parameter. 


\section{Results and discussions}

This method has several advantages with respect to the standard $\chi^{2}$-minimization technique: (I) the systematic errors can be conveniently included in the minimization procedure, without introducing a large number of additional fit parameters, (II) the error distributions of the experimental data are not assumed Gaussian a priori, but are directly evaluated from the probability distributions assigned to the data and (III) the error bands on DDPs or the differential cross section can be computed including all the correlations among the fitted parameters (see Fig. 1(b)).

The fit has been performed using two different data sets: all the available experimental data for the unpolarized cross section below pion-production threshold, denoted as FULL data set (for a total of 150 data points) and the TAPS data set (55 point) (for a detailed description of the data set see Ref. [15] and references therein). In Fig. 1(a) we show the probability distributions of the LEX coefficients of the scalar DDPs for both the data sets, while the corresponding fitted results for the DDPs as function of the $\mathrm{cm}$ photon energy $\omega$ are shown in Fig. 1(b), together with their $68 \%$ and $95 \%$ confidence level $(C L)$ uncertainty bands. The
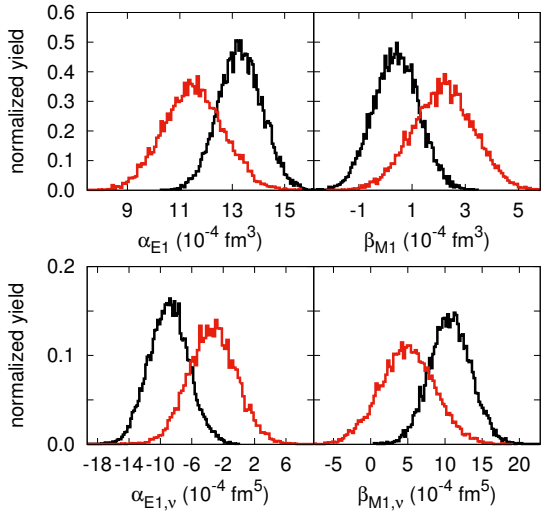

(a)

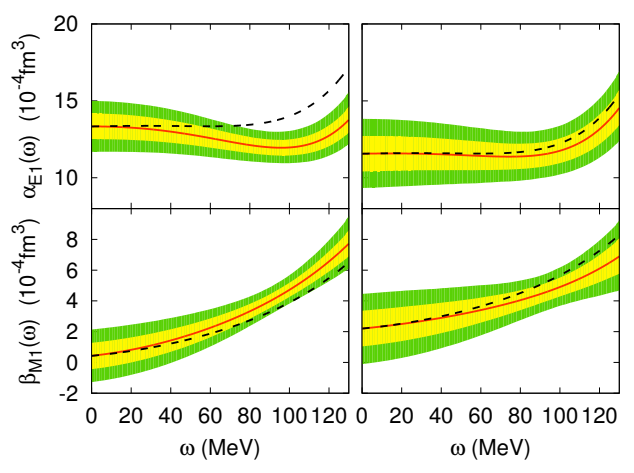

(b)

Figure 1. (a) Probability distributions of the fit parameters, with 100 bins per histogram, from the bootstrap analysis of the FULL (black lines) and TAPS (red lines) data sets: static polarizabilities $\alpha_{E 1}$ (top-left) and $\beta_{M 1}$ (top-right) and dispersive polarizabilities $\alpha_{E 1, v}$ (bottom-left) and $\beta_{M 1, v}$ (bottom-right). (b) Results from the fit of the scalar DDPs (solid line) for the FULL (left panels) and TAPS (right panels) data sets: $\alpha_{E 1}(\omega)$ on the top and $\beta_{M 1}(\omega)$ on the bottom. The $68 \%$ (yellow) and 95\% (green) $C L$ areas include all the correlations between the parameters. The dashed lines are the predictions from DRs [3].

comparison with the subtracted DR predictions (obtained with the fitted values of the static scalar dipole polarizabilities) is fairly good for both the DDPs. For $\omega \lesssim 60 \mathrm{MeV}$ the agreement is within the $68 \%$ confidence area. At higher energy, the DR predictions for $\beta_{M 1}(\omega)$ remain within the $95 \% C L$ regions for both fits with the FULL and TAPS data sets, while the DR predictions for $\alpha_{E 1}(\omega)$ show deviations from the fit results with the FULL data set and still a very good agreement (within the $68 \%$ confidence area) for the TAPS data set. The deviations observed for the FULL data sets can be a hint of inconsistencies between different data subsets. The unpolarized RCS data are more sensitive to the electric polarizability than to the magnetic one: this feature causes a larger relative error in $\beta_{M 1}(\omega)$, as clearly visible in Fig. 1(b). 


\section{Conclusions}

In summary, we discussed a new method based on the parametric bootstrap technique that allows us to extract information on the proton scalar DDPs from RCS data at low energies. The extraction of the energy dependence of the DDPs turned out to be quite challenging, because of the very low sensitivity of the unpolarized RCS data to the higher-order dispersive coefficients. This gives both large error bands of our estimates, in particular for $\beta_{M 1}(\omega)$, and strong correlations between the fit parameters.

\section{References}

[1] D. Babusci, G. Giordano, A. L'vov, G. Matone, A. Nathan, Phys. Rev. C 58, 1013 (1998)

[2] H.W. Griesshammer, T.R. Hemmert, Phys. Rev. C 65, 045207 (2002)

[3] R.P. Hildebrandt, H.W. Griesshammer, T.R. Hemmert, B. Pasquini, Eur. Phys. J. A 20, 293 (2004)

[4] V. Lensky, J. McGovern, V. Pascalutsa, Eur. Phys. J. C 75, 604 (2015)

[5] L.I. Lapidus, C.K. Chao, Sov. Phys. JETP 14, 210 (1961)

[6] V.I. Ritus, Sov. Phys. JETP 5, 1249 (1957), [ZhETP 32,1536(1957)]

[7] A.P. Contogouris, Il Nuovo Cimento 25, 104 (1962)

[8] D. Drechsel, M. Gorchtein, B. Pasquini, M. Vanderhaeghen, Phys. Rev. C 61, 015204 (1999)

[9] B. Pasquini, D. Drechsel, M. Vanderhaeghen, Phys. Rev. C 76, 015203 (2007)

[10] D. Drechsel, S.S. Kamalov, L. Tiator, Eur. Phys. J. A 34, 69 (2007)

[11] V. Olmos de Leon, et al., Eur. Phys. J. A 10, 207 (2001)

[12] P.P. Martel, et al. (A2), Phys. Rev. Lett. 114, 112501 (2015)

[13] B.R. Holstein, D. Drechsel, B. Pasquini, M. Vanderhaeghen, Phys. Rev. C 61, 034316 (2000)

[14] J.A. Nelder, R. Mead, Comput. J. 7, 308 (1965)

[15] B. Pasquini, P. Pedroni, S. Sconfietti, Phys. Rev. C 98, 015204 (2018) 